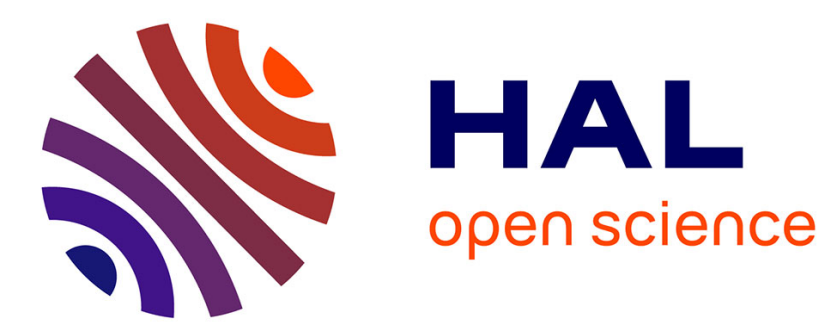

\title{
Experimental identification of turbulent fluid forces applied to fuel assemblies using an uncertain model and fretting-wear estimation
}

Anas Batou, Christian Soize

\section{- To cite this version:}

Anas Batou, Christian Soize. Experimental identification of turbulent fluid forces applied to fuel assemblies using an uncertain model and fretting-wear estimation. Mechanical Systems and Signal Processing, 2009, 23 (7), pp.2141-2153. 10.1016/j.ymssp.2009.03.018 . hal-00684339

\section{HAL Id: hal-00684339 \\ https://hal.science/hal-00684339}

Submitted on 1 Apr 2012

HAL is a multi-disciplinary open access archive for the deposit and dissemination of scientific research documents, whether they are published or not. The documents may come from teaching and research institutions in France or abroad, or from public or private research centers.
L'archive ouverte pluridisciplinaire HAL, est destinée au dépôt et à la diffusion de documents scientifiques de niveau recherche, publiés ou non, émanant des établissements d'enseignement et de recherche français ou étrangers, des laboratoires publics ou privés. 


\title{
Experimental identification of turbulent fluid forces applied to fuel assemblies using an uncertain model and fretting-wear estimation
}

\author{
A.Batou ${ }^{\mathrm{a}, \mathrm{b}}$, C.Soize $e^{*, \mathrm{a}}$ \\ ${ }^{a}$ Université Paris-Est, Laboratoire Modélisation et Simulation Multi Echelle, \\ FRE3160 CNRS, \\ 5 bd Descartes, 77454 Marne-la-Vallee, France \\ ${ }^{b}$ EDF, département Analyse Mécanique et Acoustique, \\ 1 avenue du Géneral de Gaulle, 92140 Clamart, France
}

\begin{abstract}
This paper is devoted to the identification of stochastic loads applied to fuel assemblies using an uncertain computational model and experimental measurements of responses. The stochastic loads applied to the structure are induced by a turbulent flow. The structure is made up of a nonlinear complex dynamical system. The experimental responses of the structure are obtained from strain sensors located on the structure. There are several sources of uncertainties in this experimental identification problem of the stochastic loads: uncertainties on the nonlinear dynamical computational model of the structure (fuel assemblies), uncertainties on parameters of the mathematical model of the stochastic loads themselves and finally, measurements errors. All these sources of uncertainties are identified and taken into account in the
\end{abstract}

\footnotetext{
* Corresponding author

**Principal corresponding author

Email addresses: anas.batou@univ-paris-est.fr (A.Batou), christian.soize@univ-paris-est.fr (C.Soize)
} 
identification process of the stochastic loads. Then, the stochastic nonlinear dynamical computational model of fuel assemblies on which the identified stochastic loads are applied yield interesting results concerning the robustness of the estimation of the fretting wear of the fuel rods.

Key words: identification, uncertain stochastic loads, uncertain nonlinear dynamical system, fuel assemblies.

\section{Introduction}

A fuel assembly is made up of thousands of fuel rods and tubes which are held in position by grids. This dynamical system bathes in a flow of a liquid (water)which induces turbulent forces that are likely to induce frettingwear of the fuel rods. A fuel assembly is a very complex nonlinear dynamical system for which an accurate computational model (called the reference computational model) would be time consuming and generally, would induce many numerical problems due to the high modal density of such a structure. Therefore, the computational model must be simplified from an engineering design point of view. The model uncertainties are thus due to the simplification introduced by the mathematical-mechanical modeling process. The measurements are realized with an experimental setup which is constituted of a half fuel assembly which bathes in a turbulent fluid. This experimental setup has been designed in order to improve the understanding of the dynamical behavior of fuel assemblies. The objectives of this paper are to identify the parameters of the mathematical model of the stochastic forces induced by the turbulent fluid which are applied to the experimental setup, using an uncertain stochastic simplified computational model and experimen- 
tal responses. The general methodology used to solve this problem has been presented in [2] and validated on a very simple academic example. In the present paper, we applied this methodology to a complex industrial structure and the identification of the stochastic model is carried out using measurements on an experimental setup. The identified stochastic model is then used to analyze the robustness of the predictions and allows the fretting-wear of the rods to be estimated. It should be noted that such an estimation cannot easily be performed by experiments because a very large number of cycles are required. The use of a validate stochastic model allows such an estimation to be carried out in a probabilistic context which is adapted to a reliability analysis. The uncertainties introduced in this methodology are summarized on Figure 1. In the problem under consideration, there are four sources of uncertainties:

(1) The model uncertainties induced by the introduction of simplifications in the model. This type model uncertainties are taken into account using the nonparametric probabilistic approach (see $[9,10])$ which consists in modeling the reduced mass and stiffness matrices by full random matrices defined on a probability space $(\Theta, \mathcal{T}, \mathcal{P})$. The dispersion parameters introduced by this approach will be identified using the maximum likelihood method and a reference computational model made up of an accurate finite element model.

(2) The mean model of the stochastic loads (induced by the statistical fluctuations of the turbulent pressure applied to the structure) is a vectorvalued Gaussian centered second-order stationary stochastic process defined on a probability space $\left(\Theta^{\prime}, \mathcal{T}^{\prime}, \mathcal{P}^{\prime}\right)$. Consequently, the mean model of the stochastic loads is completely defined by the nominal value of the matrix- 


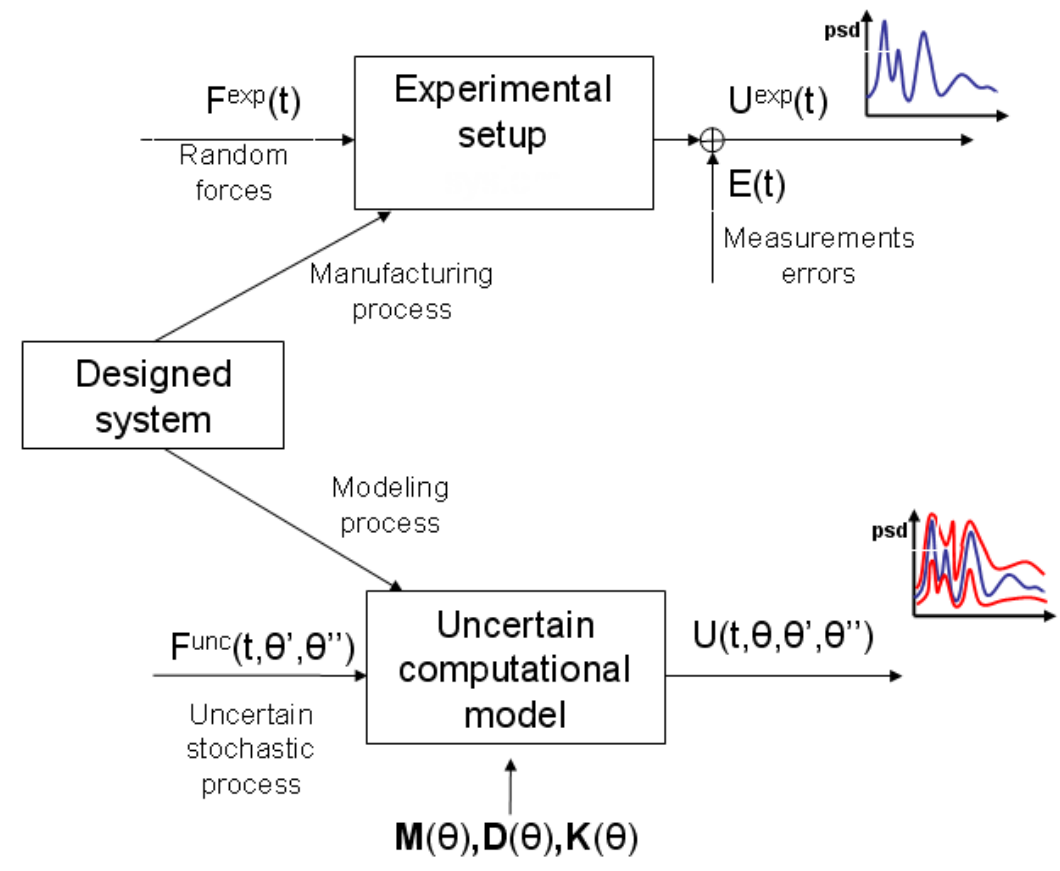

Figure 1: Designed system, experimental setup, uncertain computational model

valued spectral density function of the stochastic process. This nominal value is identified by minimizing its distance to the corresponding experimental value.

(3) The uncertainties concerning the stochastic loads are taken into account by replacing the nominal value of the matrix-valued spectral density function (defined above) by a random matrix-valued spectral density function defined on a probability space $\left(\Theta^{\prime \prime}, \mathcal{T},{ }^{\prime \prime} \mathcal{P}^{\prime \prime}\right)$. By construction, the mean value of this random matrix-valued spectral density function is equal to the nominal value of the matrix-valued spectral density function. The probability distribution of this random matrix-valued spectral density function de- 
pends on a dispersion parameter which controls the level of uncertainties and which is identified using the maximum likelihood method and experimental responses.

(4) The uncertainties induced by measurement errors are taken into account in introducing an additive random variable for the experimental observation. This random noise (modeling the effects of the measurement errors) is defined as a Gaussian centered random variable on a probability space $\left(\Theta^{\prime \prime \prime}, \mathcal{T}^{\prime \prime \prime}, \mathcal{P}^{\prime \prime \prime}\right)$.

Section 2 deals with the experimental modal analysis carried out on the experimental setup. Section 3 presents the reference computational model which will be used as an observation for the stochastic simplified computational model constructed in Section 4. The uncertain stochastic loads applied on the stochastic simplified computational model are identified in Section 5. In Section 6, the identified stochastic loads and the stochastic simplified computational model are used to construct statistics on quantities of interest, in particular the fretting-wear on the rods.

\section{Experimental measurements and modal analysis}

\subsection{Description of the experimental setup}

The experimental setup (see Figure 2) is composed of a half fuel assembly ( 4 grids instead of 8 grids). Each grid is composed of $17 \times 17$ cells (see Figure 4). The 25 guide tubes are clamped at theirs ends and soldered to the grids (see Figure 3). The 264 fuel rods are free at their ends and are held in position by bumps and springs (see Figure 3). The transversal directions are the $x$ direction and the $z$ direction. So the the longitudinal direction is the $y$ 
direction. One of the fuel rod, which is lighter than the others is located at the position J-10 on Figure 4 and is equipped with 12 strain sensors located in the two transverse directions at $0.06 \mathrm{~m}, 0.43 \mathrm{~m}, 0.69 \mathrm{~m}, 1.0 \mathrm{~m}, 1.37 \mathrm{~m}$ and $1.62 \mathrm{~m}$ from the low end of this rod. The 12 strain sensors are named $J 1 x, J 2 x, J 3 x, J 4 x, J 5 x$ and $J 6 x$ for $x$ direction and $J 1 z, J 2 z, J 3 z, J 4 z$, $J 5 z$ and $J 6 z$ for $z$ direction. All the structure bathes in a flow of a liquid (water) whose velocity is approximatively $1 \mathrm{~m} / \mathrm{s}$.
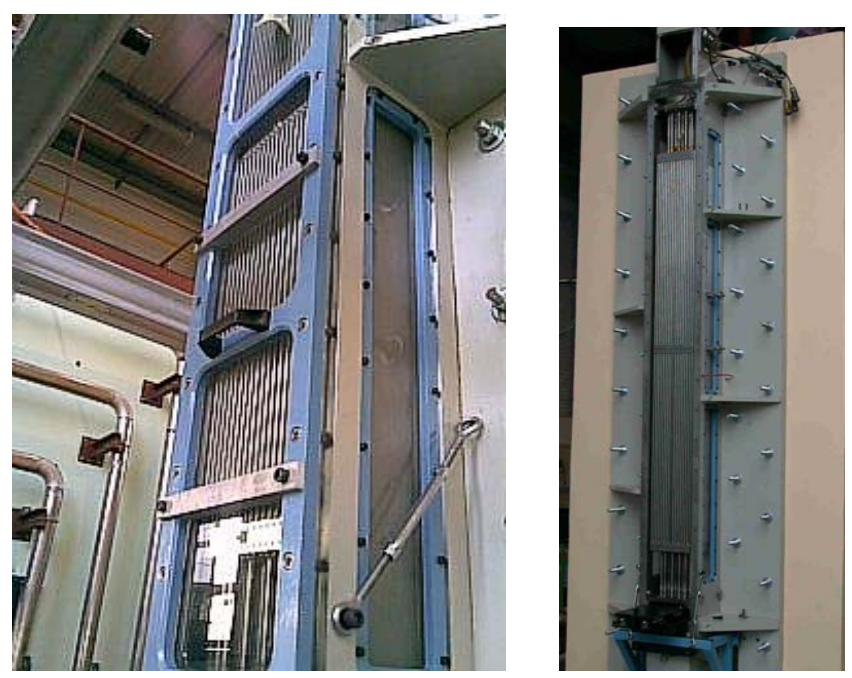

Figure 2: Experimental setup.

\subsection{Spectral density function for the measured strain.}

Only the following seven sensors are measured: $J 1 x, J 5 x, J 6 x, J 1 z$, $J 2 z, J 4 z$ and $J 6 z$. The frequency band of analysis is $\mathcal{B}=] 0,100] \mathrm{Hz}$. The maximal frequency $f_{\max }$ considered for the signal sampling is equal to $640 \mathrm{~Hz}$ for which the energy in the signals is negligible. Due to the Shannon theorem, the time step sampling is $\Delta t=0.78 \times 10^{-3} \mathrm{~s}$ and the number of acquisition 

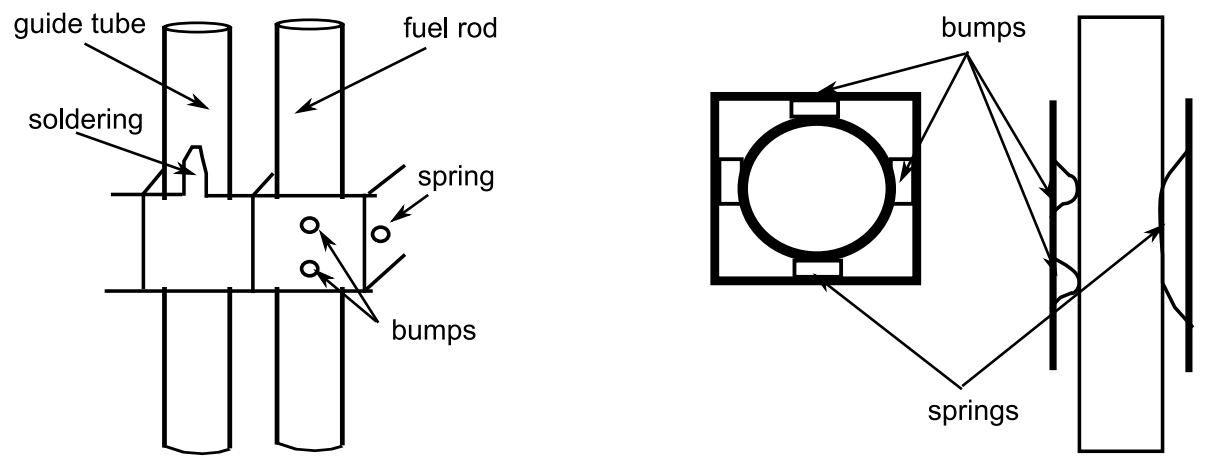

Figure 3: Grid and holding system.

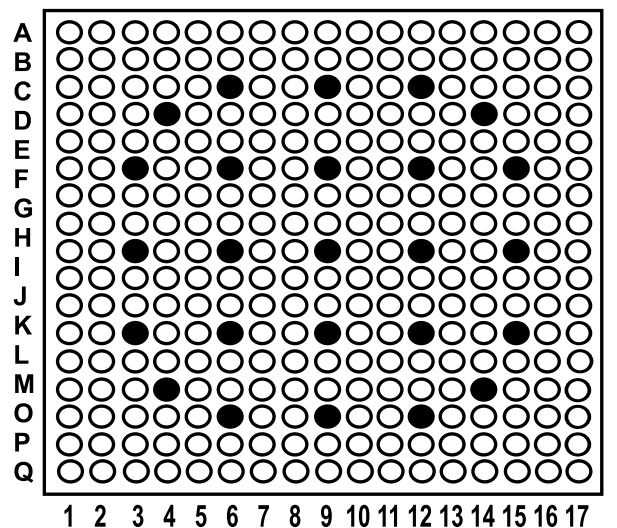

Figure 4: Grid.

points is chosen as 4096. The acquisition time is then $t_{\max }=3.2 \mathrm{~s}$. The frequency resolution is then $0.3125 \mathrm{~Hz}$. The matrix-valued spectral density function of the vector-valued measured random signal is estimated by the periodogram method (see [6]). The power spectral density functions (PSD) for sensors $J 1 x$ and $J 5 x$ are represented on Figure 5 for the frequency band of analysis. 


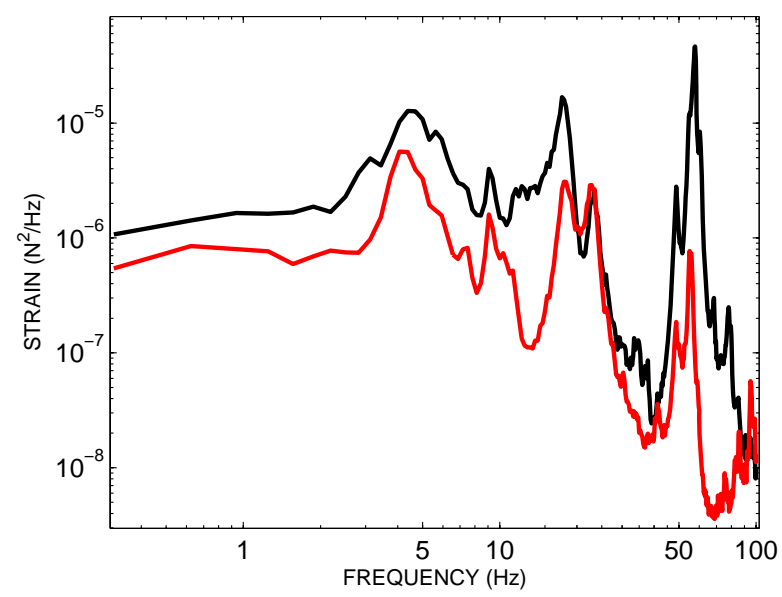

Figure 5: PSD for the strain measured by sensors $J 1 x$ (red line) and $J 5 x$ (black line).

\subsection{Experimental modal analysis}

All the eigenmodes are double eigenmodes due to the symmetries of the structure. So, the experimental modal analysis for $x$ and $z$ directions are carried out separately. It should be noted that the experimental modal masses cannot be identified because the loads induced by the fluid flow are unknown. The four first eigenfrequencies and the associated damping rates (see Table 1) correspond to the eigenmodes for which all the tubes and the rods are in phase. Note that the first eigenmode has a high damping rate.

\begin{tabular}{|l|c|c|c|c|}
\hline eigenmode & 1 & 2 & 3 & 4 \\
\hline eigenfrequency $(\mathrm{Hz})$ & 4.4 & 11,3 & 17.8 & 22.9 \\
\hline damping rate $(\%)$ & 10.1 & 5.3 & 5.3 & 3.9 \\
\hline
\end{tabular}

Table 1: Experimental eigenmodes on the frequency range $[0,30] \mathrm{Hz}$. 


\section{Reference computational model}

The reference computational model is developed in order to construct an observation which will be useful in Section 4 for the identification of the dispersion parameters $\delta_{M}^{A}$ and $\delta_{K}^{A}$ controlling the level of uncertainties in the linear subsystem of the simplified computational model. In the reference model, all the guide tubes, the fuel rods and the grids are modeled by Timoshenko's beams. The bumps and springs are modeled by springs elements. For the fuel rod equipped with sensors for measurements, the bumps and springs are modeled accurately by elastic stops (see Figure 6). The reference computational model is composed of two subsystems. The first one is linear and composed of all the guide tubes, the non-equipped fuel rods and the grids. The second one is the nonlinear fuel rod which is equipped with the sensors for measurements. The linear subsystem with free interface is analyzed and updated in the frequency band of analysis $\mathcal{B}$. In this band, there are 2502 eigenmodes. The first four eigenmodes are represented in Figure 7 and compared with the experimental values in Table 2. Those modes are ensemble modes (all the rods and tubes are in phase). The MAC criterion (see [4]) compares the experimental modal shapes and the mode shapes calculated with the reference computational model. Since there is absolutely no ambiguity to identify the four first experimental modes taking into account the separation of their eigenfrequencies, the AUTOMAC has not been used before constructing the MAC. Note that the MAC is not introduced to perform the association of experimental modes with the computed modes, but is only introduced to quantify the quality of the four first computational mode shapes with respect to the experiments. The modal density is represented 
on Figure 8 in the frequency band of analysis. It can be seen that the modal density is not homogeneous at all in the frequency band of analysis and have locally high values. This behavior of the modal density is due to the mixing of global elastic modes with a large number of local elastic modes. Such a situation induces many numerical problem for the calculation of the stationary response of the stochastic nonlinear dynamical system with random parameters and random excitation. For this reason, the reference model must be simplified from the engineering design point of view.

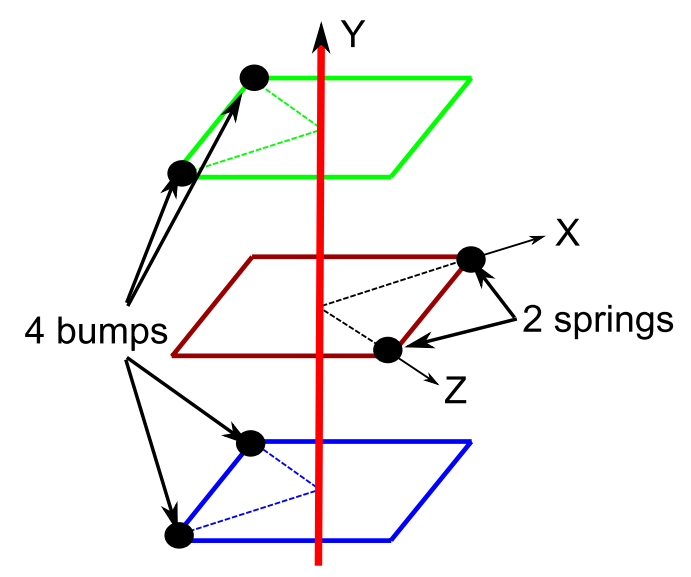

Figure 6: Elastic stops modeling.
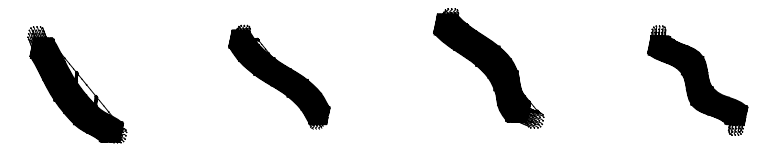

Figure 7: First four elastic modes for the reference computational model. 


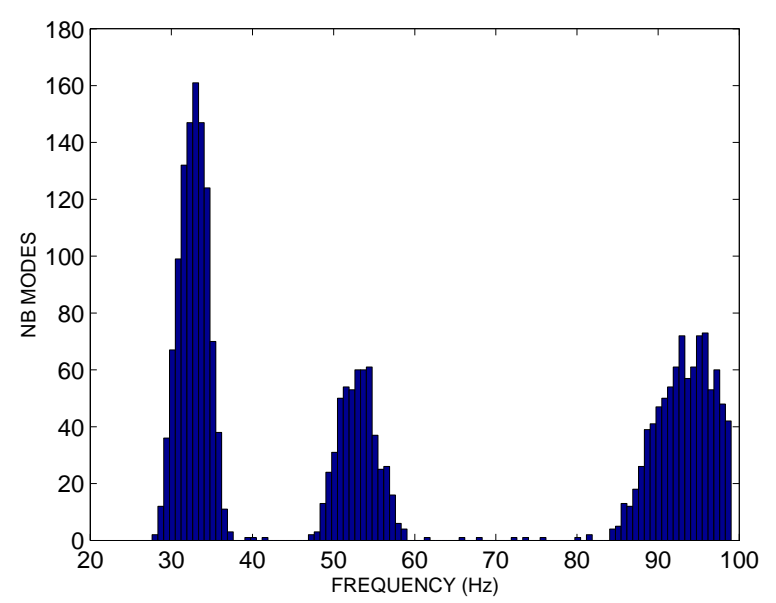

Figure 8: Modal density for the reference computational model.

\section{Simplified computational model}

\subsection{Mean simplified computational model}

The mean simplified computational model is derived from the reference computational model. Indeed, the linear subsystem of the reference computational model is replaced by an equivalent linear subsystem composed of two Timoshenko beams. The first one is equivalent to the 25 guide tubes and the other one is equivalent to the 263 non-equipped fuel rods. The nonlinear subsystem of the simplified computational model is the same as the nonlinear subsystem of the reference computational model. The simplified computational model is schematically represented on Figure 9. In the frequency band of analysis $\mathcal{B}$, there are 27 eigenfrequencies for the linear subsystem with free interface. The first four eigenmodes are represented on Figure 10 and compared with the experimental values in Table 2 . The linear subsystem and the linear part of the nonlinear subsytem are reduced using the Craig \& 
Bampton method (see [3]).

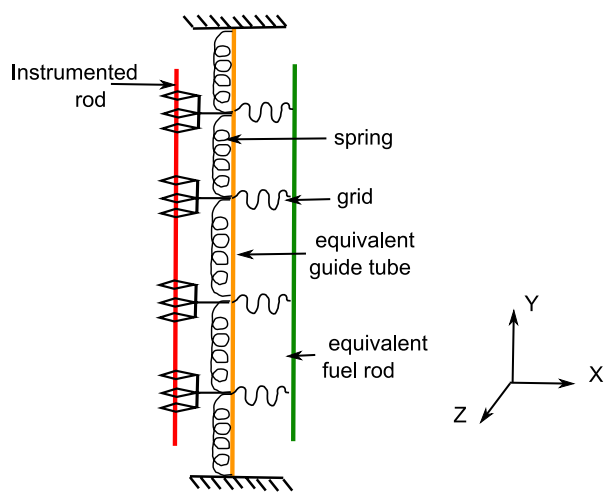

Figure 9: Simplified computational model.
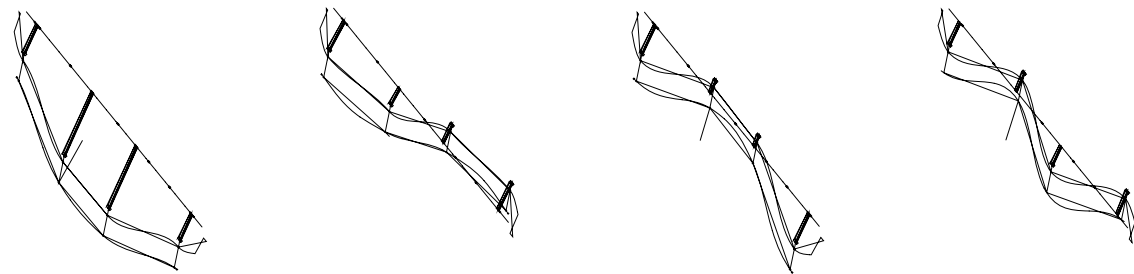

Figure 10: First four elastic modes for the simplified computational model.

\subsection{Stochastic simplified computational model}

The simplifications introduced in the simplified computational model induce model uncertainties which have to be taken into account. In [2], the model uncertainties on the linear subsystem of the reference computational model are taken into account using the nonparametric probabilistic approach. For the linear subsystem, this method consists in replacing the reduced mass 


\begin{tabular}{|c|c|c|c|c|}
\hline eigenmode & 1 & 2 & 3 & 4 \\
\hline experimental eigenfrequency (Hz) & 4.4 & 11.3 & 17.8 & 22.9 \\
\hline reference m. eigenfrequency (Hz) & 4.35 & 10.9 & 15.8 & 22.3 \\
\hline MAC ref/exp (\%) & 0.96 & 0.74 & 0.95 & 0.91 \\
\hline simplified m. eigenfrequency (Hz) & 4.34 & 10.5 & 16.3 & 23.7 \\
\hline MAC simpl/exp (\%) & 0.95 & 0.62 & 0.82 & 0.81 \\
\hline
\end{tabular}

Table 2: Comparison of the reference, the simplified and the experimental models.

and the reduced stiffness matrices of the mean reduced simplified computational model by random matrices defined on the probability space $(\Theta, \mathcal{T}, \mathcal{P})$. The probability density functions of these full random matrices depend on the dispersion parameters $\delta_{M}^{A}$ and $\delta_{K}^{A}$ (the superscript $A$ is relative to the linear subsystem). The vector $\boldsymbol{\delta}=\left(\delta_{M}^{A}, \delta_{K}^{A}\right)$ is identified introducing the random variable $J(\boldsymbol{\delta})$ defined by (see [2])

$$
J(\boldsymbol{\delta})=\int_{\mathcal{B}}\left\|\left[\mathbf{Z}^{A}(\omega)\right]^{-1}\right\|_{F}^{2} d \omega
$$

where $\|\cdot\|_{F}$ is the Frobenius norm such that $\|A\|_{F}^{2}=\operatorname{tr}\left\{[A]^{*}[A]\right\}$, with $[A]^{*}=[\bar{A}]^{T},[\bar{A}]$ is the conjugate of $[A]$ and $\operatorname{tr}$ is the trace for matrices. The matrix $\left[\mathbf{Z}^{A}(\omega)\right]$ corresponds to the condensation of the random dynamical stiffness matrix of the linear subsystem on the interface between the linear subsystem and the nonlinear one. This random variable quantifies the dynamical effects of the linear subsystem on the nonlinear one. The deterministic variable $J^{r e f}$ is constructed using the reference computational model. The parameter $\boldsymbol{\delta}$ is identified using the maximum likelihood method (see [8]) for the random variable $J(\boldsymbol{\delta})$ for which the value $J^{\text {ref }}=1.53 \times 10^{-9}$ is one realization. The graph of function $\left(\delta_{M}^{A}, \delta_{K}^{A}\right) \mapsto p_{J}\left(J^{r e f} ;\left(\delta_{M}^{A}, \delta_{K}^{A}\right)\right)$ is plotted 
on Figure 11. The maximum is reached for $\boldsymbol{\delta}^{o p t}=(0.13,0.4)$. The stationary stochastic response $\mathbf{U}(t)$ of the stochastic simplified computational model (for the complete uncertain nonlinear dynamical system excited with an uncertain stochastic loads) is such that $\mathbf{U}(t)=[H] \mathbf{Q}(t)$ where the projection matrix $[H]$ is constructed using the Craig \& Bampton substructuring technique for the two subsystems. The stationary stochastic process $\mathbf{Q}(t)$ is a vector whose components are the physical DOF at the coupling interface and are the generalized DOF for the two subsystems with fixed coupling interface. For all fixed $t$, the random variable $\mathbf{Q}(t)$ satisfies the random differential equation

$$
[\mathbf{M}] \ddot{\mathbf{Q}}(t)+[D] \dot{\mathbf{Q}}(t)+[\mathbf{K}] \mathbf{Q}(t)+\mathcal{F}^{N L}(\mathbf{Q}(t), \dot{\mathbf{Q}}(t))=\mathcal{F}(t)
$$

in which $\dot{\mathbf{Q}}(t)$ and $\ddot{\mathbf{Q}}(t)$ are the velocity and the acceleration. In this equation, $[D]$ is a deterministic matrix and $[\mathbf{M}]$ and $[\mathbf{K}]$ are random matrices. Vector $\mathcal{F}(t)$ is the generalized uncertain stochastic loads due to the turbulent flow. Vector $\mathcal{F}^{N L}(\mathbf{Q}(t), \dot{\mathbf{Q}}(t))$ is the generalized localized nonlinear forces due to the elastic stops. The detailed construction of the different terms in Eq. (2) can be found in [2].The stochastic equation (2) is solved using the Monte Carlo simulation method (see [7]).

\section{Identification of the uncertain stochastic loads}

The uncertain stochastic loads induced by the turbulent flow are applied to the four grids in $x$ and $z$ directions. So there are 8 stochastic forces which are modeled by a vector-valued stochastic process $\left\{\tilde{\mathbf{F}}^{\text {unc }}(t), t \in \mathbb{R}\right\}$. For the construction of stochastic process $\tilde{\mathbf{F}}^{\text {unc }}$, first we introduce a stochastic pro- 


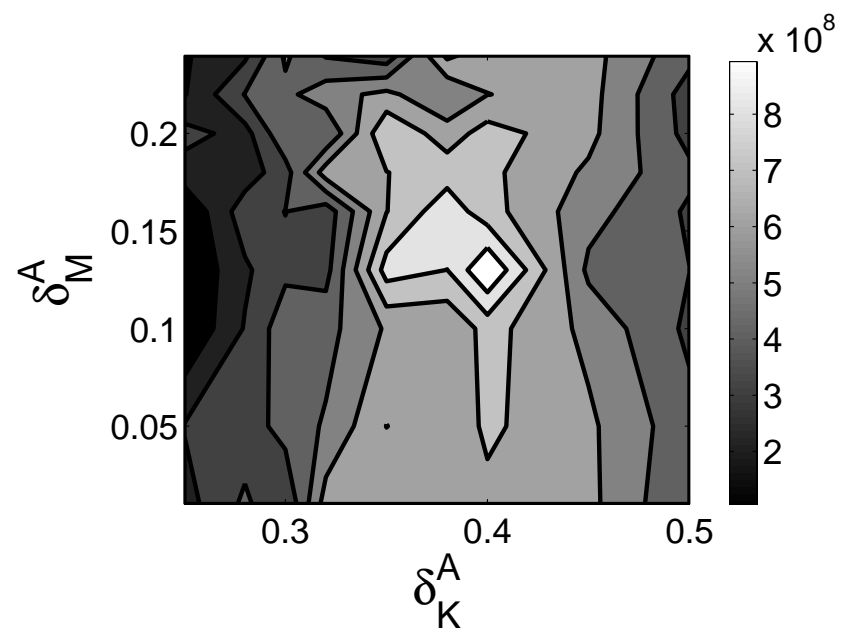

Figure 11: Graph of the function $\left\{\left(\delta_{M}^{A}, \delta_{K}^{A}\right) \mapsto p_{J}\left(J^{r e f} ;\left(\delta_{M}^{A}, \delta_{K}^{A}\right)\right)\right\}$.

cess $\{\tilde{\mathbf{F}}(t), t \in \mathbb{R}\}$ of the stochastic loads without uncertainties. It is then assumed that stochastic process $\tilde{\mathbf{F}}$ is a Gaussian stationary centered secondorder stochastic process defined on a probability space $\left(\Theta^{\prime}, \mathcal{T}^{\prime}, \mathcal{P}^{\prime}\right)$ for which the matrix-valued spectral density function is $\left\{\left[S_{\tilde{\mathbf{F}}}(\omega)\right], \omega \in \mathbb{R}\right\}$. The uncertain stochastic process $\tilde{\mathbf{F}}^{\text {unc }}$ is then constructed as the stochastic process $\tilde{\mathbf{F}}$ for which the deterministic function $\left\{\left[S_{\tilde{\mathbf{F}}}(\omega)\right], \omega \in \mathbb{R}\right\}$ is replaced by a random function $\left\{\left[\mathbf{S}_{\tilde{\mathbf{F}}}(\omega)\right], \omega \in \mathbb{R}\right\}$ defined on a probability space $\left(\Theta^{\prime \prime}, \mathcal{T}^{\prime \prime}, \mathcal{P}^{\prime \prime}\right)$. The probability distribution of random function $\left\{\left[\mathbf{S}_{\tilde{\mathbf{F}}}(\omega)\right], \omega \in \mathbb{R}\right\}$ is constructed using the maximum entropy principle and depends on a dispersion parameter $\delta_{F}$. It is proven in [2] that such a stochastic process is stationary, centered, second-order but not Gaussian. It is also proven that the matrix-valued spectral density function $\left\{\left[S_{\tilde{\mathbf{F}}^{\text {unc }}}(\omega)\right], \omega \in \mathbb{R}\right\}$ of the uncertain stochastic process $\tilde{\mathbf{F}}^{\text {unc }}$ is in fact equal to the function $\left\{\left[S_{\tilde{\mathbf{F}}}(\omega)\right], \omega \in \mathbb{R}\right\}$. Such a stochastic process and its generator of independent realizations are completely defined 
by (1) the spectral density function $\left[S_{\tilde{\mathbf{F}}^{\text {unc }}}\right]$ and (2) its dispersion parameter $\delta_{F}$. So the identification of the stochastic loads consists in identifying these two quantities.

5.1. Identification of the matrix-valued spectral density function of the uncertain stochastic loads

The first step consists in identifying a rough approximation of $\left[S_{\tilde{\mathbf{F}}^{\text {unc }}}\right]$ in order to construct a simplified algebraic representation of $\left[S_{\tilde{\mathbf{F}}^{\text {unc }}}\right]$. For that, the inverse method proposed by Granger in [5] is applied in order to construct such a rough approximation of the matrix-valued function $\left[S_{\tilde{\mathbf{F}}^{\text {unc }}}(\omega)\right]$. Figure 12 shows the rough approximation obtained for the PSD of the four forces following $z$ direction. As it can be seen in this figure (in log-log representation), the simplified algebraic representation of the PSD can be constructed in choosing a constant part of a given amplitude $A$ in the frequency band $\left[0, \omega_{0}\right]$ with $\omega_{0}=2 \pi f_{0}, f_{0}=28 \mathrm{~Hz}$ and a linearly decreasing part for $\omega>\omega_{0}$ for which the angle is $\alpha$. Therefore, each PSD $S_{i}(\omega), i=1, \ldots, 8$, can be parameterized as follow: (1) for $\omega \leq \omega_{0}, S_{i}(\omega)=A_{i}$, (2) for $\omega>\omega_{0}$, $S(\omega)=A_{i}\left(\omega / \omega_{0}\right)^{\alpha}$ with $\alpha=1.4$ (in this simplified algebraic model, it is assumed that angle $\alpha$ is a fixed constant). Moreover, we suppose that the amplitudes $A_{i}, i=1, \ldots, 8$ are constant following $y$ direction. Consequently, the simplified algebraic representation of the spectral density function of the uncertain stochastic loads depends only on the two parameters $A_{X}^{G}$ and $A_{Z}^{G}$ which are the amplitudes of the PSD in each transversal direction. The identification of the spectral density function of the uncertain stochastic loads then consists in identifying the vector $\mathbf{r}=\left(A_{X}^{G}, A_{Z}^{G}\right)$. We introduce the vector-valued stochastic process $\left\{\boldsymbol{\Xi}^{\exp }(t), t \in \mathbb{R}\right\}$ whose components are the 


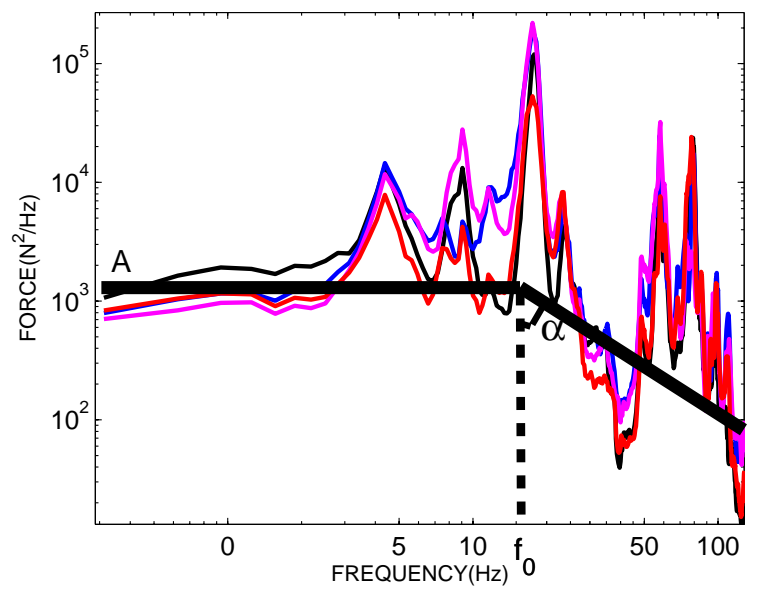

Figure 12: Approximation for the PSD for the forces applied to grid 1 (black), grid 2 (blue), grid 3 (purple) and grid 4 (red). Parametric representation for the function [S $\left.S_{\tilde{\mathbf{F}}^{\text {unc }}}\right]$ (thick dark line).

7 measured strains for which the matrix-valued spectral density function $\left\{\left[S_{\boldsymbol{\Xi}^{\mathrm{exp}}}(\omega)\right], \omega \in \mathbb{R}\right\}$ is estimated using the periodogram method. The corresponding stochastic process $\{\boldsymbol{\Xi}(t ; \mathbf{r}), t \in \mathbb{R}\}$ is calculated with the stochastic simplified computational model. The matrix-valued spectral density function $\left\{\left[S_{\Xi}(\omega ; \mathbf{r})\right], \omega \in \mathbb{R}\right\}$ of the stochastic process $\boldsymbol{\Xi}(t ; \mathbf{r})$ is also estimated using the periodogram method. The identification is then performed by minimizing the distance between the experimental matrix-valued spectral density function $\left[S_{\Xi^{\exp }}(\omega)\right]$ and the numerical matrix-valued spectral density function $\left[S_{\Xi}(\omega ; \mathbf{r})\right]$. The optimal value $\mathbf{r}_{\text {opt }}$ of the parameter $\mathbf{r}$ is then given by

$$
\mathbf{r}_{\text {opt }}=\arg \min _{\mathbf{r} \in \mathcal{C}_{r}} D(\mathbf{r}) \quad, \quad D(\mathbf{r})=\int_{\mathcal{B}}\left\|\left[S_{\boldsymbol{\Xi}}(\omega ; \mathbf{r})\right]-\left[S_{\boldsymbol{\Xi}^{\mathrm{exp}}}(\omega)\right]\right\|_{F}^{2} d \omega,
$$


in which $\mathcal{C}_{r}$ is the admissible set for the vector $\mathbf{r}$. In Figure 12, it can be seen that the initial value of $\mathbf{r}$ can be chosen as $1 /(2 \pi) \times\left(10^{3}, 10^{3}\right) N^{2} /(\mathrm{rad} / \mathrm{s})$. Function $D(\mathbf{r})$ is represented in Figure 13. The minimal value of $D$ is reached for $\mathbf{r}_{\text {opt }}=1 /(2 \pi) \times\left(2.8 \times 10^{3}, 2.0 \times 10^{3}\right) N^{2} /(\mathrm{rad} / \mathrm{s})$.

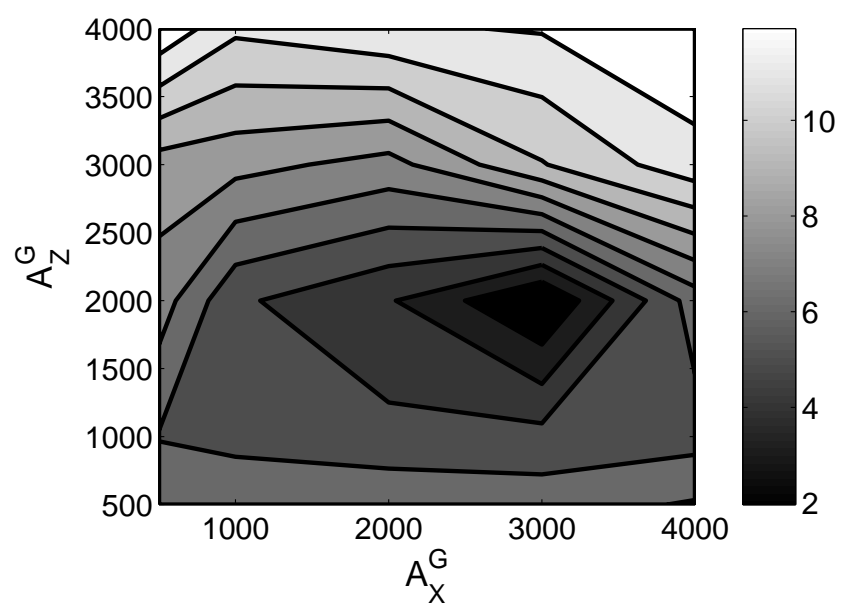

Figure 13: Graph of function $\mathbf{r} \mapsto D(\mathbf{r})$.

\subsection{Identification of the dispersion parameter $\delta_{F}$.}

The dispersion parameter $\delta_{F}$ is identified using the maximum likelihood method for the random variable $J_{s}$ which is such that for all $\theta \in \Theta$ and for all $\theta^{\prime \prime} \in \Theta^{\prime \prime}$,

$$
J_{s}\left(\theta, \theta^{\prime \prime}\right)=\int_{\mathcal{B}}\left\|\left[\mathbf{S}_{\Xi}\left(\omega, \theta, \theta^{\prime \prime}\right)\right]\right\|_{F}^{2} d \omega
$$

and for which the experimental value $J_{s}^{\exp }$ defined by

$$
J_{s}^{\exp }=\int_{\mathcal{B}}\left\|\left[S_{\Xi^{\exp }}(\omega)\right]\right\|_{F}^{2} d \omega
$$


is one realization. We then have the following optimization problem

$$
\delta_{F}^{o p t}=\arg \max _{\delta_{F} \in \mathcal{C}_{\delta_{F}}} p_{J_{s}}\left(J_{s}^{\text {exp }} ; \delta_{F}\right),
$$

where $\delta_{F}^{o p t}$ is the optimal value for $\delta_{F}$. The function $\delta_{F} \mapsto p_{J_{s}}\left(J_{s}^{\exp } ; \delta_{F}\right)$ is represented on Figure 14. The maximum is reached for $\delta_{F}^{o p t}=0.40$.

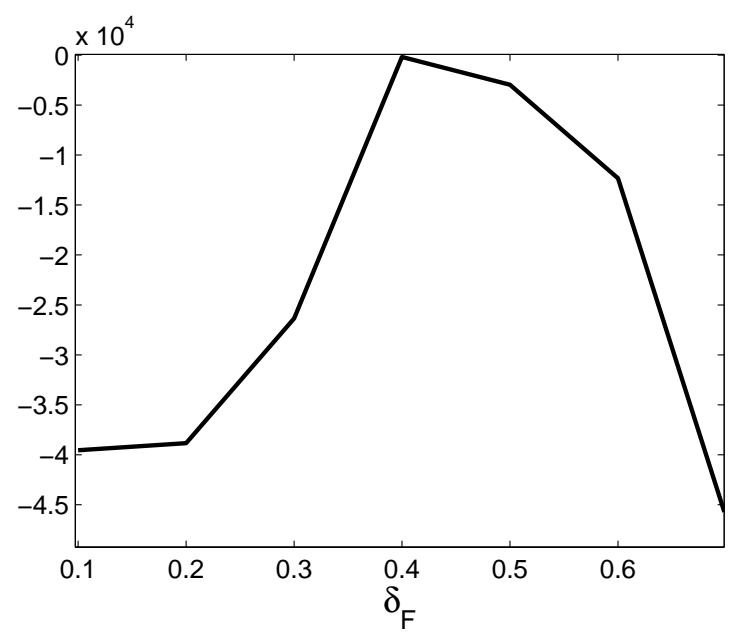

Figure 14: Graph of function $\delta_{F} \mapsto p_{J_{s}}\left(J_{s}^{\exp } ; \delta_{F}\right)$.

\subsection{Measurements errors}

The measurements errors on the variable $J_{s}^{\exp }$ defined by Eq. (5) are modeled by an additive noise $\mathcal{E}$, defined on a probability space $\left(\Theta^{\prime \prime \prime}, \mathcal{T}^{\prime \prime \prime}, \mathcal{P}^{\prime \prime \prime}\right)$, for which the probability density function is $e \mapsto p_{\mathcal{E}}(e)$. We then have

$$
J^{\exp }=J_{s}^{\text {true }}+\mathcal{E}
$$


where the variable $J_{s}^{\text {true }}$ is the true experimental value of the variable $J_{s}$ (without measurements errors). The random variable $J_{s}^{\text {er }}$ corresponding to the random variable $J^{\exp }$ and calculated by the stochastic simplified computational model is then such that

$$
J_{s}^{\mathrm{er}}=J_{s}+\mathcal{E}
$$

where the random variable $J_{s}$ is defined by Eq. (4). Then, the probability density function $y \mapsto p_{J_{s}^{\text {er }}}(y)$ of the random variable $J_{s}^{\text {er }}$ is defined by

$$
p_{J_{s}^{\mathrm{er}}}(y)=\int_{-\infty}^{+\infty} p_{J_{s}^{\mathrm{er}} \mid \mathcal{E}=e}(y \mid e) p_{\mathcal{E}}(e) d e
$$

where $x \mapsto p_{J_{s}^{\mathrm{er}} \mid \mathcal{E}=e}(y \mid e)$ is the conditional probability density function $J_{s}^{\mathrm{er}}$ given $\mathcal{E}=e$ which is, using Eq. (8), such that

$$
p_{J_{s}^{\mathrm{er}} \mid \mathcal{E}=e}(y \mid e)=p_{J_{s}}(y-e) .
$$

The probability density function $y \mapsto p_{J_{s}^{\mathrm{er}}}(y)$ of the random variable $J_{s}^{\mathrm{er}}$ depends on $\delta_{F}$ and is rewritten as

$$
p_{J_{s}^{\text {er }}}\left(y ; \delta_{F}\right)=\int_{-\infty}^{+\infty} p_{J_{s}}\left(y-e ; \delta_{F}\right) p_{\mathcal{E}}(e) d e
$$

It is assumed that the additive noise $\mathcal{E}$ is modeled by a centered Gaussian random variable. For the experimental setup, the measurements errors on the strain are about 10\%. In this condition, the value of the standard deviation of $\mathcal{E}$ is chosen as $J_{s}^{\exp } / 10$. Then the dispersion parameter $\delta_{F}$ is identified 
using the maximum likelihood method and we then have

$$
\delta_{F}^{o p t}=\arg \max _{\delta_{F} \in \mathcal{C}_{\delta_{F}}} p_{J_{s}^{\mathrm{er}}}\left(J_{s}^{\mathrm{exp}} ; \delta_{F}\right)
$$

where $\delta_{F}^{o p t}$ is the optimal value for $\delta_{F}$. The integral in Eq. (11) is estimated using the Monte Carlo simulation method. Generating $\nu_{e}$ independent realizations $e_{1}, \ldots, e_{\nu_{e}}$ of the random variable $\mathcal{E}$ with p.d.f $p_{\mathcal{E}}(e)$, the value of $p_{J_{s}^{\text {er }}}\left(J_{s}^{\exp } ; \delta_{F}\right)$ is estimated by

$$
p_{J_{s}^{\text {er }}}\left(J_{s}^{\exp } ; \delta_{F}\right)=\frac{1}{\nu_{e}} \sum_{i=1}^{\nu_{e}} p_{J_{s}}\left(J_{s}^{\exp }-e_{i} ; \delta_{F}\right)
$$

The graph of function $\delta_{F} \mapsto p_{J_{s}^{\text {er }}}\left(J_{s}^{\exp } ; \delta_{F}\right)$ is plotted in Figure 15. The optimal value for the dispersion parameter is $\delta_{F}^{\text {opt }}=0.30$. This value of $\delta_{F}^{\text {opt }}$ is lower than the value estimated in Section 5.2.

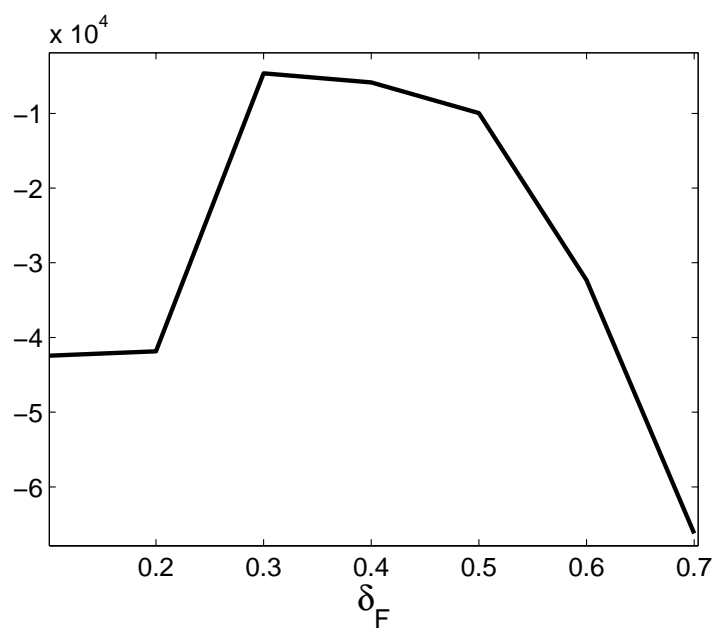

Figure 15: Graph of function $\delta_{F} \mapsto p_{J_{s}^{\mathrm{er}}}\left(J_{s}^{\exp } ; \delta_{F}\right)$. 


\section{Stochastic response of the stochastic simplified model submitted}

to the identified uncertain stochastic loads

In this section, we present an experimental validation of the identified stochastic simplified model submitted to the identified uncertain stochastic loads. The confidence region of the random function $\omega \mapsto\left\{\left(\theta, \theta^{\prime \prime}\right) \mapsto\right.$ $\left.\left[\mathbf{S}_{\Xi}\left(\omega, \theta, \theta^{\prime \prime}\right)\right]_{j j}\right\}$ (random PSD) is calculated for a probability level $P_{c}=0.95$ for sensors $J 1 x, J 5 x$ and $J 6 x$ corresponding to $j=1,2$ and 3 respectively. The comparison of this computational prediction with the experimental responses is given in Figures 16 to 18.

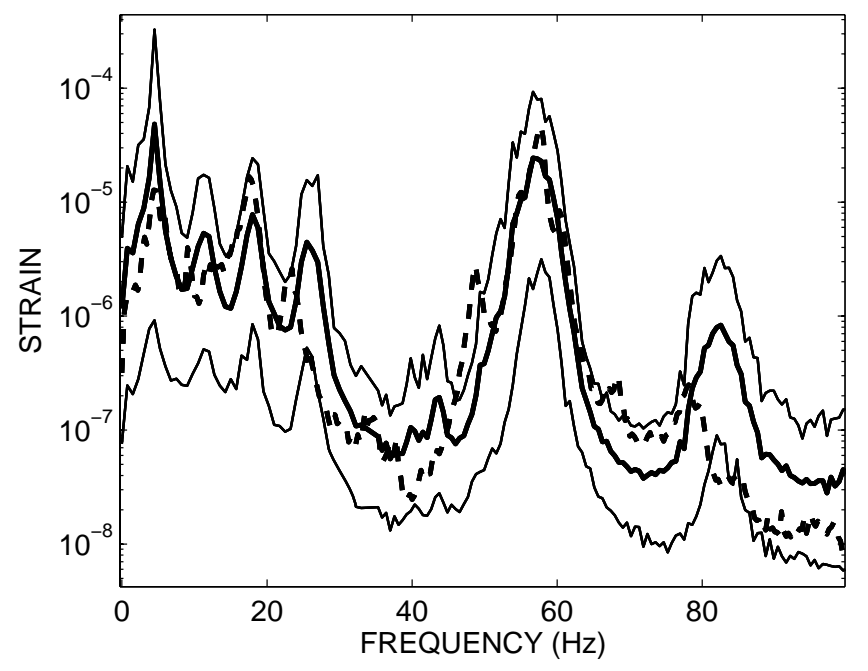

Figure 16: For sensor $J 1 x$, PSD for the stochastic strain: upper and lower envelopes and mean response (solid lines); experimental strain (dashed line).

The convergence of the estimation is represented in Figure 19 which shows the graph of function $\nu \mapsto \frac{1}{\nu} \sum_{i=1}^{\nu} J_{s}\left(\theta_{i}, \theta_{i}^{\prime \prime}\right)$. The parameter $\nu$ represents the number of independent realizations of the random mass and stiffness 


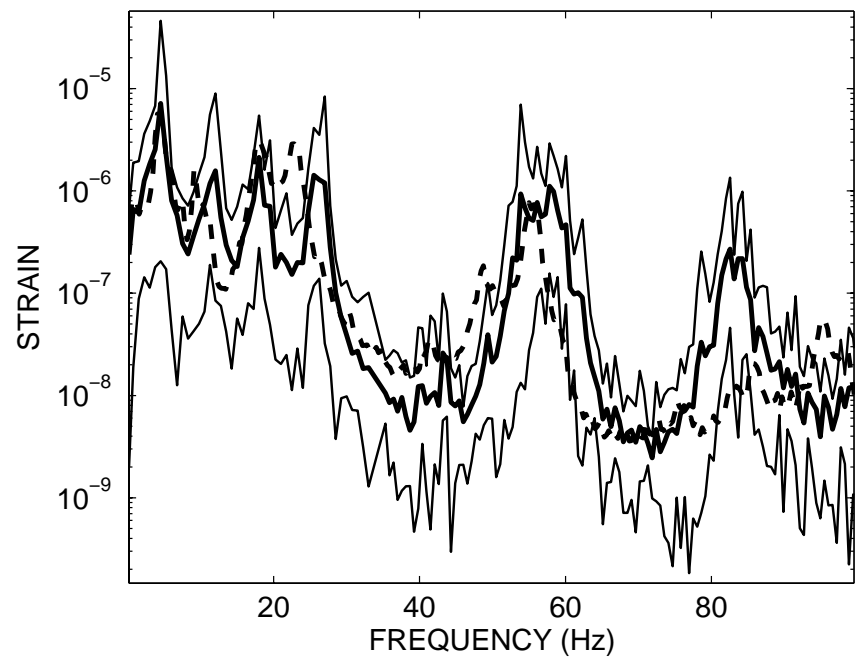

Figure 17: For sensor $J 5 x$, PSD for the stochastic strain: upper and lower envelopes and mean response (solid lines); experimental strain (dashed line).

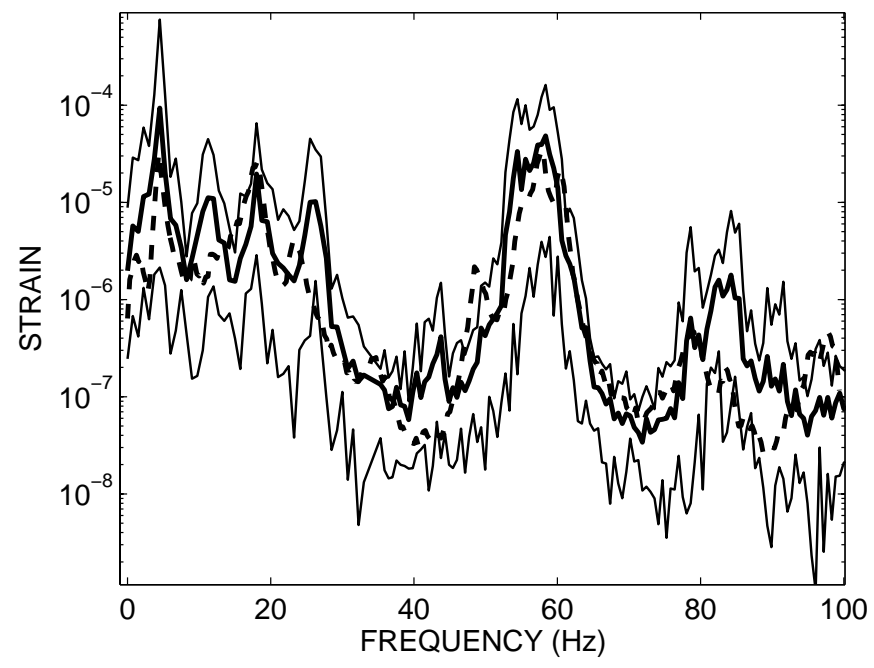

Figure 18: For sensor $J 6 x$, PSD for the stochastic strain: upper and lower envelopes and mean response (solid lines); experimental strain (dashed line). 


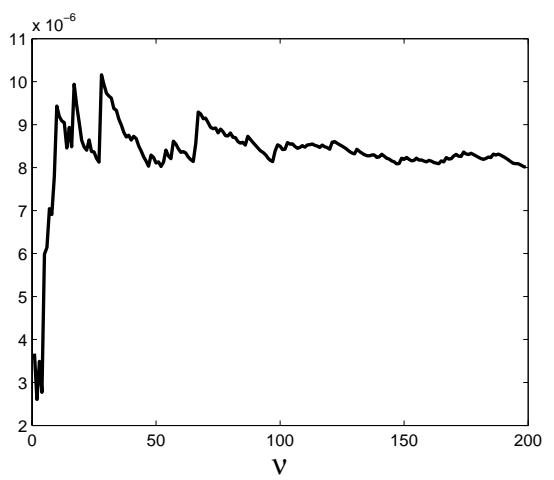

Figure 19: Graph of function $\left\{\nu \mapsto 1 / \nu \sum_{i=1}^{\nu} J_{s}\left(\theta_{i}, \theta_{i}^{\prime \prime}\right)\right\}$.

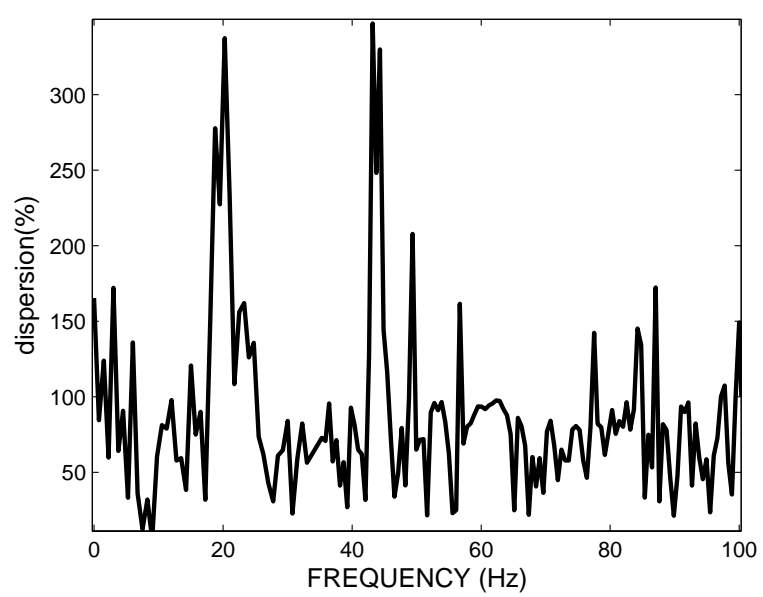

Figure 20: Graph of the dispersion of the random variable $\left(\theta, \theta^{\prime \prime}\right) \mapsto\left[\mathbf{S}_{\boldsymbol{\Xi}}\left(2 \pi f, \theta, \theta^{\prime \prime}\right)\right]_{11}$ for sensor $J 1 x$ as a function of the frequency $f$.

matrices and of the random function $\left[\mathbf{S}_{\tilde{\mathbf{F}}}\right]$. The random variable $J_{s}$ is defined by Eq. (4).

For each $\omega$, the dispersion (ratio of the standard deviation with the mean value) of the random variable $\left(\theta, \theta^{\prime \prime}\right) \mapsto\left[\mathbf{S}_{\boldsymbol{\Xi}}\left(\omega, \theta, \theta^{\prime \prime}\right)\right]_{11}$ for sensor $J 1 x$ is presented in Figure 20. The dispersion fluctuates around $70 \%$ but increases 
until $300 \%$ for some frequencies.

The random fretting-wear in the contacts rod/bump and rod/spring for the uncertain system submitted to the uncertain stochastic excitation is based on the use of the Archard power wear (see [1]) and is defined as the random variable $\left(\theta, \theta^{\prime \prime}\right) \mapsto P_{u s}\left(\theta, \theta^{\prime \prime}\right)$ such that

$$
P_{u s}\left(\theta, \theta^{\prime \prime}\right)=\int_{\Theta^{\prime}} F_{N}\left(t, \theta, \theta^{\prime}, \theta^{\prime \prime}\right) V_{T}\left(t, \theta, \theta^{\prime}, \theta^{\prime \prime}\right) d \mathcal{P}\left(\theta^{\prime}\right)
$$

where $F_{N}(t)$ is the random normal force and where $V_{T}(t)$ is the absolute value of the random tangential velocity. It should be noted that $P_{u s}\left(\theta, \theta^{\prime \prime}\right)$ is independent of $t$ because the stochastic processes $\left\{F_{N}(t), t \in \mathbb{R}\right\}$ and $\left\{V_{T}(t), t \in \mathbb{R}\right\}$ are stationary. The mean value, the dispersion (ratio of the standard deviation with the mean value), and quantiles $5 \%$ and $95 \%$ of the random fretting-wear for the first grid following $x$ direction are reported in Table 3. The estimated dispersions are lower than $61 \%$.

\begin{tabular}{|c|c|c|c|c|}
\hline & mean & dispersion & quantile 5 \% & quantile 95 \% \\
\hline low bump & 0.024 & $60.1 \%$ & 0.014 & 0.035 \\
\hline spring & 0.029 & $51.7 \%$ & 0.025 & 0.036 \\
\hline high bump & 0.014 & $55.4 \%$ & 0.01 & 0.019 \\
\hline
\end{tabular}

Table 3: Statistics for the random fretting-wear on the first grid following $x$ direction.

\section{Conclusions}

We have presented a complete methodology for the identification of turbulent fluid forces applied to fuel assemblies using an uncertain simplified computational model and experimental strain responses. All the sources of 
uncertainties have been taken into account in the identification process. The probabilistic model of model uncertainties in the simplified computational model depends on dispersion parameters which have been identified using the maximum likelihood method and a reference computational model. The uncertainties concerning the parametric representation of the uncertain stochastic loads have also been taken into account. The uncertain stochastic loads have been identified taking into account measurements errors. The identified stochastic loads have been applied to the stochastic simplified computational model in order to construct the statistics on the random fretting-wear of the fuel roads. The estimated dispersions of the random fretting-wear are about

$60 \%$ that induces a relatively robustness with respect to uncertainties for this complex industrial problem.

\section{References}

[1] J.F. Archard, Elastic deformation and the laws of friction, Proceeding of the royal society of London, serie A243, 10-205 (1957).

[2] A. Batou, C. Soize, Identification of stochastic loads applied to a non-linear dynamical system using an uncertain computational model and experimental responses, Computational Mechanics, 43(4), 559-571 (2009).

[3] R.R. Craig, Jr. and M.C.C Bampton, Coupling for substructures for dynamic analysis, AIAA Journal, 3 (6), July 1968.

[4] D.J. Ewins, Modal Testing: theory and practice, Research Study Press LTD, John Wiley and Sons, 1984. 
[5] S. Granger and L. Perotin, An inverse method for the identification of a distributed random excitation acting on a vibrating structure, part 1: theory, Mechanical System and Signal Processing, 13 (1), 53-65, 1999.

[6] M.B. Priestley, Spectral Analysis and Time Series, Academic Press, New York, 1981.

[7] R.Y. Rubinstein, Simulation and the Monte Carlo Method, John Wiley and Sons, 1980.

[8] R.J. Serfling, Approximation Theorems of Mathematical Statistics, John Wiley \& Sons , New York 1980.

[9] C. Soize, A nonparametric model of random uncertainties for reduced matrix models in structural dynamics, Probabilistic Engineering Mechanics, 15(3) (2000)277-294.

[10] C. Soize, Random matrix theory for modeling uncertainties in computational mechanics, Computer Methods in Applied Mechanics and Engineering, 194 (2005) 1333-1366. 